\title{
Simultaneous Determination of Synthetic Colorants in Pickles and Sauerkrauts by HPLC-MS
}

\author{
Pei Yao, Shu-Bai Li ${ }^{\text {a }}$, Yuan Liu and Qi-Meng Zhang \\ Changzhou Vocational Institute of Engineering, Changzhou, PR China \\ asbli@email.czie.net (Corresponding author)
}

Keywords: High performance liquid chromatography-mass spectrometry/mass spectrometry; synthetic colorants; pickles and sauerkrauts.

Abstract:The Objective of this paper is to establish a method for determination of six synthetic colorants (sunset yellow, tartrazine, amaranth, carmine, allura red, brilliant blue)in pickles and sauerkrauts. The samples were adsorbed by polyamide then desorbed by aqueous alkali. After purified and extracted, the samples were detected by reverse phase liquid chromatography using mobile phase of methanol and acetic ammonium solution (the concentration is $0.02 \mathrm{~mol} / \mathrm{L}$ ), mixture under gradient elution, confirmed and quantified by mass spectrum in the selected reaction monitoring (SRM) mode via positive electrospray ionization $(\mathrm{ESI}+)$. The results show that the method of sample testing had strong qualitative and quantitative ability. The curve of peak area with concentration was from 0.1 to $50 \mathrm{mg} / \mathrm{L}$ with correlation coefficients from 0.9990 to 0.9999 . The detection limit of these six synthetic colorants was between 0.4 and $13 \mu \mathrm{g} / \mathrm{kg}$. The recovery of the method was from $89.0 \%$ to $101.2 \%$, and the relative standard deviation (RSD) was between $0.5 \%$ and $1.6 \%$. The method developed is sensitive, simple, accurate and can be used to qualify and quantify this six synthetic colorants in pickles and sauerkrauts.

\section{Introduction}

The source of food colorants can be divided into natural pigments and synthetic colorants in two categories, including synthetic colorant, which has been used widely with its bright colors and low price. However, synthetic colorants were made from aniline in coal tar as raw material, which has been demonstrated to cause negative effects on human health in case of overdose. Therefore, all countries in the world have a strict control on the scope and quantity of use. At present, the main method for detection of synthetic colorants were high performance liquid chromatography[1,2,3,4], thin layer chromatography[5,6], oscillopolarography[7]. Most of these methods are low sensitivity, interference and so on in determination of colorants. Especially the composition in pickled vegetables is complex, many interference factors exist, it is easier to make misjudgment and omission of judgment. Some researcher made some analysis methods together[8,9]. So the method is established in this paper to determine six synthetic colorants in pickles and sauerkrauts simultaneously. It is used with High performance liquid chromatography-mass spectrometry and mass spectrometry at the same time, which can solve the problems in the above methods.

\section{Materials and Experimental Methods}

Chemicals and Apparatus Methanol and ammonium acetate were purchased from Tedia(American International Chemical, Inc. California, USA).their purity were Chromatographic Pure . Sunset yellow, tartrazine, amaranth, carmine, allura red, brilliant blue standards were purchased from the national standard material research center. Methanoic acid, ammonia water, ethanol were purchased from GuangHua Company(Guangzhou, China). All of these reagents were analytically pure. Polyamide was purchased from GuangHua Company (Guangzhou, China). The size of powder: 100-200 mesh. Water was purified using a Milli-Q Ultra-pure water purification system (Millipore, Bedford, MA, USA). 
Triple quadrupole mass spectrometer, America's Quantum Access quaternary gradient pump, Accela, automatic sampler, and Xcalibur workstation were purchased from Thermo Fisher Company (USA). Ultra high performance liquid chromatograph (HPLC)and mass spectrometer (MS) were purchased from UPLC Waters Company (USA), Waters Symmetry C18 chromatographic column $(50 \mathrm{~mm} \times 2.1 \mathrm{~mm}, 1.9 \mu \mathrm{m})$ was purchased from Waters Company (USA). $0.22 \mu \mathrm{m}$ nylon filter was got from Pall Corporation(USA).All other reagents were analytically pure.

Experiment Condition of HPLC and MS Thermo Hypersil GOLD $(50 \mathrm{~mm} \times 2.1 \mathrm{~mm}, 1.9 \mu \mathrm{m})$ column were used. The Column temperature was maintained at $30^{\circ} \mathrm{C}$. The injected sample volume was $10 \mu \mathrm{l}$. The flow-rate used was $200 \mu \mathrm{L} / \mathrm{min}$.. The mobile phase was a mixture of methanol and 0.02M ammonium acetate, and a gradient elution method was used. The optimized chromatographic condition was: $0 \sim 2 \mathrm{~min}$, the concentration of methanol is $15 \%$; $2 \sim 3 \mathrm{~min}$, linear variation of methanol is $98 \%$, keeping time was $7 \mathrm{~min}, 10 \sim 11 \mathrm{~min}$, linear variation of methanol is $15 \%$, keeping time was $4 \mathrm{~min}$. Then return to initial conditions; a $10 \mathrm{~min}$ post-run time was used after each analysis. The column was conditioned prior to use by washing with Methanol and deionizes water successively.

Table 1 Choice reaction monitoring conditions of six kinds of colorants

\begin{tabular}{ccccc}
\hline Item & Parent ion(m/z) & Daughter ion(m/z) & Collision energy(V) & $\begin{array}{c}\text { Lens } \\
\text { compensation(V) }\end{array}$ \\
\hline sunset yellow & 409 & 173 & 20 & 69 \\
tartrazine & 469 & 136 & 22 & 72 \\
& & 451 & 23 & 106 \\
amaranth & 539 & 200 & 20 & 142 \\
& & 348 & 27 & \\
carmine & 539 & 223 & 38 & 97 \\
allura red & 453 & 158 & 25 & 154 \\
& & 217 & 26 & \\
brilliant blue & \multirow{2}{*}{} & 202 & 31 & \\
& & 171 & 48 & 47 \\
\hline
\end{tabular}

This HPLC system was connected to a mass spectrometer (HPLC Waters Company ,USA), equipped with an electrospray interface operating in negative ion, using the following operation parameters: capillary voltage $3500 \mathrm{~V}$, electro spray ionization(ESI), in positive mode, selected reaction monitoring $(\mathrm{SRM})$, resolution ratio: $\mathrm{Q} 1=0.7 \mathrm{M}, \mathrm{Q} 3=0.7 \mathrm{M}$, Sheath gas $\mathrm{N}_{2}$, pressure 39 au, Aux gas $\mathrm{N}_{2}$, pressure $10 \mathrm{au}$. Heat the temperature of the capillary: $350^{\circ} \mathrm{C}$, Collision gas pressure, $1.5 \mathrm{~m}$ Torr, sweep length(Scan Width $\mathrm{m} / \mathrm{z}$ ) 0.100, scanning time is $0.200 \mathrm{~s}$. Other conditions are shown in the Table 1.Choice ion current chromatograms of 6 kinds of colorants are shown in Fig.1.

Experimental Method Stirring pickles and sauerkrauts into pieces in a blender, about $5.00 \mathrm{~g}$ (accurately weighed to $0.01 \mathrm{~g}$ ) of samples were weighed into a $30 \mathrm{ml}$ neck flask and dissolved with water, adjust $\mathrm{pH}$ to 6 by citric acid. $1.00 \mathrm{~g}$ (accurately weighed to $0.01 \mathrm{~g}$ ) of polyamide powder and a little water were mixed together uniformity then added to the solution of samples, ultrasound extracted for 15mins, suction filtration by G3 sintered funnel, washed 3 5 times through water, then washed 3 5 times by mixture of methanol and methanoic acid, then washed until neutral by water, desorbed 3 5 times by mixture of alcohol and ammonia water, $5 \mathrm{ml}$ each time, collect desorbing liquid, added acetic acid to neutral, vapored to almost dry, add water to solve. Finally, the neck flask was constant volume to $50 \mathrm{ml}$ with water. After being shaken and centrifuged, colorants were collected through a filter with pore size of $0.22 \mu \mathrm{m}$ before injection into the HPLC system. 

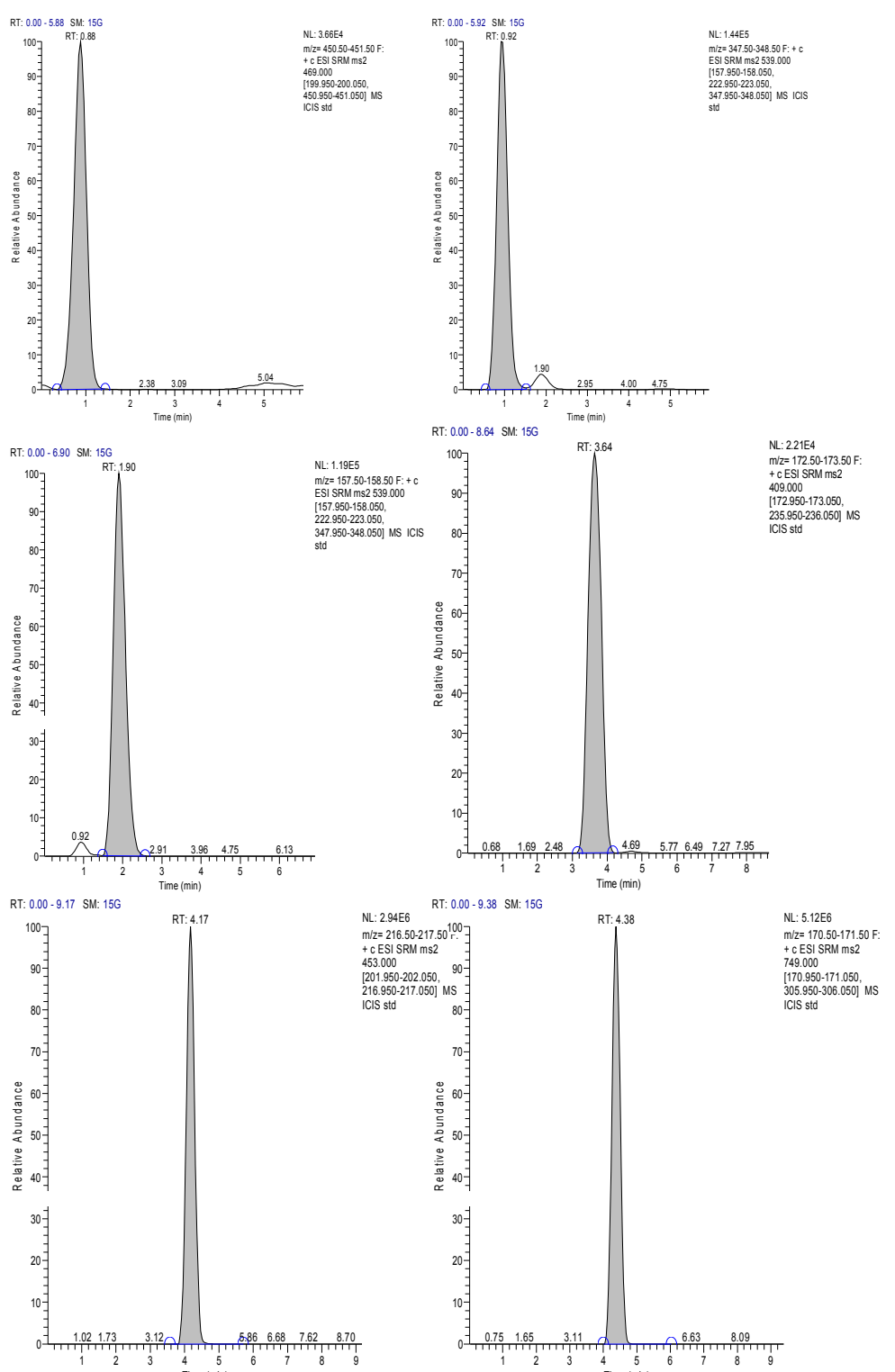

Fig. 1 Choice ion current chromatograms of ${ }^{\text {Trmetmin }} 6$ kinds of colorants

\section{Result and Discussion}

Choice Of Chromatographic Condition Using methanol and ammonium acetate $(0.02 \mathrm{M})$ as mobile phase, then gradient eluted and isocratic elution individually, the results show that not only acquired satisfied peak and degree of separation ,but also reduced the time of analysis and the noise of instrument.

Calibration Curve Recovery Rate and Precision When the concentration between 0.1 and $50 \mathrm{mg} / \mathrm{L}$, The linear relation between peak area Y concentrate X of six synthetic colorants are good. The calibration curve of these synthetic colorants were shows in table 2 . The results shows that the linearity of the analytical 
Table 2 The equation of linear regression and correlation coefficient of the six synthetic colorants

\begin{tabular}{ccc}
\hline Components name & regression equation & $\mathrm{R}$ \\
\hline Tartrazine & $\mathrm{Y}=1599.1 \mathrm{X}+221$ & 0.9990 \\
Amaranth & $\mathrm{Y}=5879.8 \mathrm{X}+106$ & 0.9995 \\
Carmine & $\mathrm{Y}=5674.7 \mathrm{X}+331$ & 0.9993 \\
Sunset yellow & $\mathrm{Y}=1273.2 \mathrm{X}+138$ & 0.9991 \\
Allura red & $\mathrm{Y}=8162.9 \mathrm{X}+445$ & 0.9996 \\
Brilliant blue & $\mathrm{Y}=14128.9 \mathrm{X}+507$ & 0.9999 \\
\hline
\end{tabular}

response within the studied range of two orders of magnitude is excellent, with correlation coefficients higher than 0.9990 in all case.

Add $5.00 \mathrm{mg}$ (accurately weighed to $0.01 \mathrm{~g}$ ) of these synthetic colorants into a blank sample. After disposing, parallel test were done and calculate recovery rate, recovery and precision are showed in Table 3.All results showed that recovery rate was from $89.0 \%$ to $101.2 \%$. The relative standard deviation (RSD) was between $0.5 \%$ and $1.6 \%$.

Table 3 Recovery rate and precision for the 6 kinds of synthetic colorants

\begin{tabular}{lcccccc}
\hline Item & $\begin{array}{c}\text { Sunset } \\
\text { Yellow }\end{array}$ & Tartrazine & Amaranth & Carmine & Allura red & Brilliant blue \\
& & & & & & 5.06 \\
Estimated value & 4.63 & 4.78 & 4.94 & 4.45 & 4.66 & 101.2 \\
Recovery (\%) & 92.6 & 95.6 & 98.8 & 89.0 & 93.2 & 0.6 \\
RSD (\%) & 1.6 & 1.0 & 0.8 & 1.9 & 1.1 & \\
\hline
\end{tabular}

Samples Test To evaluate the effectiveness of the proposed method, it was applied to the analysis of a total of 18 samples of pickles and sauerkrauts in market randomly. Fortunately, in most cases, most synthetic colorants were not found in the samples. Unfortunately, the results indicate that four samples which added sunset yellow is illegally. Testing results of samples were shown in Table 4 .

Table 4 Testing results of all samples (the concentration unit is $\mathrm{g} / \mathrm{kg}$ )

\begin{tabular}{lcccccc}
\hline Sample & sunset yellow & tartrazine & amaranth & carmine & allura red & brilliant blue \\
\hline Sample1 & 0.002 & ND & 0.004 & ND & ND & ND \\
Sample2 & ND & ND & 0.008 & ND & ND & ND \\
Sample3 & ND & ND & ND & ND & ND & ND \\
Sample4 & 0.003 & ND & 0.003 & 0.006 & ND & ND \\
Sample5 & 0.003 & ND & 0.003 & ND & ND & ND \\
Sample6 & ND & ND & ND & ND & ND & ND \\
Sample7 & ND & ND & 0.008 & ND & ND & ND \\
Sample8 & ND & ND & ND & ND & ND & ND \\
Sample9 & ND & ND & ND & ND & ND & ND \\
Sample10 & 0.004 & ND & 0.004 & 0.008 & ND & ND \\
Sample11 & 0.003 & ND & 0.007 & ND & ND & ND \\
Sample12 & ND & ND & 0.011 & ND & ND & ND \\
Sample13 & ND & ND & ND & 0.007 & ND & ND \\
Sample14 & 0.003 & ND & ND & ND & ND & ND \\
Sample15 & 0.004 & ND & ND & ND & ND & ND \\
Sample16 & ND & ND & ND & ND & ND & ND \\
Sample17 & 0.005 & ND & 0.003 & 0.008 & ND & ND \\
Sample18 & ND & ND & ND & ND & ND & ND \\
\hline
\end{tabular}

$\mathrm{ND}$ : it is not detected in sample.

\section{Conclusion}

This paper established a method of a high-performance liquid chromatography-mass spectrometry/mass spectrometry determination six synthetic colorants (lemon yellow, amaranth carmine, sunset yellow fcf, allura Red AC, brilliant blue) in pickles and sauerkrauts. The method of sample testing had strong qualitative and quantitative ability, fully applied in determination of samples. This is a real example that illustrates the useful determination for six synthetic colorants with the HPLC/MS simultaneously. The method developed is sensitive, simple, accurate and suitable 
for the determination of six synthetic colorants in pickles and sauerkrauts simultaneously. It provided practical technical means to monitor synthetic colorants in pickles and sauerkrauts. It is important significance in the use of food additives. The method is rapid and has been shown to be reliable.

\section{Acknowledgements}

This work was supported by Colleges and universities in Jiangsu province of outstanding young teachers and principals of overseas training program, Jiangsu Qinglan Project, and Fund of JiangSu Student Innovation Training Program.

\section{References}

[1] B.R. Li, J.L. Feng, Z.Q. Pan, et al. Chin J Health Lab. Technol. Vol.17(2007),p.579

[2] B. Saad, M.F. Bari, S. M. Idiris, et al: Chromatography A Vol. 1073(2005), p. 393

[3] C. Ripolles, E. Pitarch,J.V. Sancho, et al:Analyt. Chim. Acta Vol.682(2011), p.102

[4] L.P. Ruan, W.L. Ji, H.L. Liu, et al. Chin J Food Hyg Vol. 23(2011) ,p.151

[5] Z.F. Zou, M. Pu, J.J. Li, et al. Chin J Food Hyg. Vol.22(2010),p.112

[6] Y.X. Gu, Y. Ge, J. Yin, et al. Food Ferm. Ind.Vol. 38(2012),p.161

[7] M. Ma, X. Luo, B. Chen, et al. J Chromatography A Vol. 103(2006); p.170

[8] K.S. Miniotia, C.F. Sakellariou, N.S. Thomaidis, et al.Analyt. Chim. Act. Vol.583(2007),p. 103

[9] D.J. Li, M.Z. Zhao, G.J. Luan. Food Res. Dev. Vol. 25(2004),p. 107 